\title{
Reductions in Complex Mismatch Negativity to Extra Tone Gestalt Pattern Deviance in First-Episode Schizophrenia
}

OPEN ACCESS

Edited by:

Kiyoto Kasai,

University of Tokyo, Japan

Reviewed by:

Tsuyoshi Araki,

University of Tokyo, Japan

Minah Kim,

Seoul National

University Hospital,

South Korea

*Correspondence:

Dean F. Salisbury

salisburyd@upmc.edu

${ }^{\dagger}$ Present address:

Sarah M. Haigh,

Department of Psychology, University of Nevada - Reno,

Reno, NV, United States

Specialty section: This article was submitted to

Schizophrenia,

a section of the journal

Frontiers in Psychiatry

Received: 25 February 2020

Accepted: 18 May 2020

Published: 08 June 2020

Citation:

Salisbury DF, Coffman BA and Haigh SM (2020) Reductions in Complex Mismatch Negativity to Extra

Tone Gestalt Pattern Deviance in

First-Episode Schizophrenia.

Front. Psychiatry 11:505. doi: 10.3389/fpsyt.2020.00505

\author{
Dean F. Salisbury*, Brian A. Coffman and Sarah M. Haigh ${ }^{\dagger}$ \\ Clinical Neurophysiology Research Laboratory, Department of Psychiatry, Western Psychiatric Hospital, University of \\ Pittsburgh School of Medicine, Pittsburgh, PA, United States
}

Although "simple" mismatch negativity (sMMN) to stimulus parameter changes is robustly reduced in long-term schizophrenia (Sz), it is much less reduced in individuals at their first psychotic episode in the schizophrenia-spectrum (FESz). "Complex" MMN (cMMN) reflecting pre-attentive acoustic pattern analysis is also markedly reduced in Sz, but is little studied in FESz. The computational complexity of pattern analysis reflected in cMMN may more greatly stress auditory processing, providing a more sensitive measure of auditory processing deficits at first break. If so, cMMN would provide information about the underlying pathophysiology early in disease course, and may serve as a biomarker for pathology in the Sz prodrome. Twenty-two FESz individuals were compared to 22 volunteer healthy controls $(\mathrm{HC})$ on SMMN and CMMN tasks. For SMMN, pitch- and duration-deviants were presented among standard repetitive tones. For cMMN, repeated groups of 3 identical tones were presented with occasional (14\%) groups including an extra identical 4th tone deviant. FESz did not show reductions of pitchdeviant (Cohen's $d=0.08$ ) or duration-deviant MMNs ( $d=-0.02)$, but showed large reduction in extra-tone cMMN $(d=0.83)$. Reduced cMMN was associated with poor social functioning. Reduction in $\mathrm{CMMN}$ but not in sMMNs in FESz suggests impairments in late perceptual pattern processing. CMMN is sensitive to subtle pathology and functioning early in disease course which may, in turn, impact social functioning. Future studies in clinical high risk individuals are needed to determine whether this putative biomarker of disease presence is sensitive to the prodromal stage of schizophrenia.

Keywords: mismatch negativity, schizophrenia, first episode psychosis, social functioning, biomarker

\section{INTRODUCTION}

The auditory mismatch negativity (MMN), a neurophysiological measure related to automatic deviance detection, has engendered much research in clinical neuroscience to understand the basic pathophysiology of schizophrenia due to its severe impairment in long-term schizophrenia (Sz). The MMN elicited by infrequent changes in acoustic parameters, such as pitch-deviants (pMMN) and duration-deviants (dMMN), show effect sizes (Cohen's d) in Sz between 0.8 and $1.2(1,2)$. 
However, this simple parameter MMN (sMMN) is not as reduced in individuals at their first episode of schizophrenia (FESz) (3-6). Recent meta-analyses suggest a dMMN effect size in FESz of 0.4 to $0.5(2,7)$ (which dropped to 0.36 when only studies that matched premorbid IQ or education were included) and a negligible effect size for $\mathrm{pMMN}$ of $<0.04$. Thus, sMMN does not appear to be as sensitive to the pathophysiology early in disease course as it is in later stages.

Like in FESz, sMMN reductions have been, at best, equivocal in clinical high risk for psychosis (CHR) individuals and in truly prodromal individuals within the $\mathrm{CHR}$ state. Despite some promising reports of greater $\mathrm{dMMN}$ reductions in $\mathrm{CHR}$ individuals that converted to psychosis $(8,9)$, replication has been difficult. For example, Patricia Michie's group originally reported smaller $\mathrm{dMMN}$ in a group of $6 \mathrm{CHR}$ individuals that later transitioned to psychosis (10), but recently reported no dMMN reduction in $7 \mathrm{CHR}$ individuals who transitioned to psychosis; in fact those who transitioned to psychosis evinced larger $\mathrm{dMMN}$ than those that did not (11). There have also been reports of $\mathrm{dMMN}$ being larger in CHR individuals, e.g., (12), although these were very young individuals. In Erickson et al's meta-analysis (2), CHR studies showed an effect size of $d=0.4$, again a relatively small effect, with close to chance probability that a CHR individual selected at random would show a MMN worse than the healthy mean (61\%).

Thus, sMMN reductions appear to be smaller in early psychosis. Given that sMMN is quite reduced in long-term illness, is related to the underlying circuit pathophysiology (13), and shows progressive reductions after psychosis onset that correlate with longitudinal gray matter loss (5), it is presumed that such auditory pathophysiology is present to a lesser degree early in the psychosis course and prior to the emergence of psychosis. If so, a more computationallydemanding MMN measure may be sensitive to this subtle early disease course pathophysiology. While different etiologies with different pathophysiology may be related to the symptom heterogeneity in schizophrenia at first episode, nearly all patients will show MMN reduction after a few years of psychosis (5). In that most FESz individuals have little sMMN reduction at first episode but develop deficits with disease course, there appears to be a common auditory pathology associated with sMMN across possible differential etiologies. If so, it is possible that a variation of the standard mismatch task may reveal subtle pathology to which $\mathrm{SMMN}$ is insensitive.

Healthy individuals produce MMN to a variety of deviants in complex patterns (hereby referred to as complex MMN; cMMN) including descending-pitch tone pairs among standard ascending-pitch tone pairs (14-19), repeated tones among a pattern of alternating tones (20), rules that predict the duration or pitch of the next tone (21), missing tones in groups of identical tones (22), and extra tones that were identical to the other tones in a group (23), among other patterns. Rather than relying on an infrequent physical change among frequent identical sounds, $c M M N$ depends on the abstraction of some sort of pattern rule. Complex auditory pattern tasks may elicit a later deviance-related negativity (i.e.,
cMMN) than the sMMN. Several studies have reported multiple MMNs, including the late $\mathrm{cMMN}$, in response to a complex pattern deviant (24-28). We hypothesize that the later, presumably more computationally-demanding cMMN is impacted earlier in psychotic disease course.

Complex auditory pattern analysis and cMMN have been examined in long-term illness. Alain et al (20) found no significant difference in $\mathrm{cMMN}$ to a repeat deviant in longterm schizophrenia, although there was a trend-level group difference when the nose electrode was used as the reference. The cMMN to a missing tone on a gestalt grouping task was significantly reduced in long-term schizophrenia (29) and in early-course schizophrenia (30). The cMMN to infrequent extra tones in a gestalt grouping task is reduced in long-term schizophrenia (23). Descending pitch deviants among ascending pitch paired tones produced a smaller cMMN in those with long-term schizophrenia (31). The cMMN to tones that violate an ascending pitch pattern, and to tones that violate a musical scale pattern are reduced in schizophrenia (32). Thus, like sMMN, cMMN appears to be robustly reduced in long-term schizophrenia. However, cMMN tends to have a longer rather than later latency than sMMN, the similarities and differences in neural substrate between sMMN and cMMN are not known, and it has rarely been examined in close proximity to the emergence of psychosis (at first episode or earlier).

Having validated complex auditory pattern protocols as eliciting reduced cMMN in long-term illness, we have begun testing them in FESz individuals. Although descending pitch deviants among ascending pitch paired tones showed reduced cMMN in long-term schizophrenia, tone-pair cMMN was within normal limits in FESz (31). In the current study, we measured $\mathrm{MMN}$ to violations in an extra-tone gestalt grouping rule in $\mathrm{SZ}$ and in FESz, modified from a protocol validated to elicit reduced cMMN in Sz (23). Further, sMMN (pMMN and dMMN) were examined to compare effect sizes between various paradigms.

Although sMMN may not be highly sensitive to pathophysiology in early disease course, functional decline spans several domains (and predates the onset of overt psychosis). Keshavan and colleagues (personal communication, November 18,2016$)^{1}$ coined the acronym CLAASSIC for this progression, representing deficits first in Cognition and Learning, then problems in Affect and increased Anxiety, followed by Social deficits, and finally a progression from Subthreshold to Intermittent to Chronic psychotic symptoms. Similarly, Cornblatt et al. (33) coined the acronym CASIS for a progression of Cognitive deficits followed by Affective disturbance, Social dysfunction, increased Isolation, and finally increasing School and work impairments. Cornblatt et al (34) reported that social and role functioning were more impaired individuals later transitioning to psychosis compared to individuals that did not transition to psychosis. Thus, in addition to examination of cMMN amplitudes in FESz, we examined the degree to which $\mathrm{cMMN}$ reductions correlated with social deficits at first clinical contact.

${ }^{1}$ At the Annual Pittsburgh Schizophrenia Conference. 


\section{METHODS}

\section{Participants}

Twenty-two FESz recruited from consecutive admissions to Western Psychiatric Institute and Clinic (WPIC) inpatient and outpatient services were compared with 22 healthy controls (HC). Fourteen FESz were diagnosed with schizophrenia (paranoid $=11$, undifferentiated $=3$ ), 2 with schizoaffective disorder (depressed subtype), 5 with psychotic disorder NOS, and 1 with schizophreniform disorder. FESz participated within two months of their first clinical contact for a first episode of psychosis, and had less than 2 months of lifetime antipsychotic medication exposure. Five FESz (22.7\%) were unmedicated.

All subjects had normal hearing as assessed by audiometry, at least nine years of schooling, and an estimated IQ over 85. None of the participants had a history of concussion or head injury with sequelae, history of alcohol or drug addiction, detox in the last 5 years, or neurological comorbidity. Groups were matched for age, gender, premorbid estimates of intelligence based on the Wechsler Abbreviated Scale of Intelligence (WASI) IQ, and parental socioeconomic status. The 4-factor Hollingshead Scale was used to measure socioeconomic status (SES) in participants and in their parents. FESz evinced trend-level lower SES than $\mathrm{HC}$, consistent with social and occupational impairment as a disease consequence (see Table $\mathbf{1}$ for demographic measures). All participants provided informed consent, and were paid for participation. All procedures were approved by the University of Pittsburgh IRB.

\section{Diagnostic Assessments}

Diagnosis was based on the Structured Clinical Interview for DSM-IV (SCID-P). Symptoms were rated using the Positive and Negative Symptom Scale (PANSS), Scale for Assessment of Positive Symptoms (SAPS), and Scale for Assessment of Negative Symptoms (SANS). All tests were conducted by an expert diagnostician (see Table $\mathbf{1}$ for clinical measures).

TABLE 1 | Demographic and clinical information.

\begin{tabular}{lccc}
\hline & FESz & HC & p \\
\hline N (F) & $22(7)$ & $22(9)$ & .53 \\
Age (sd) & $22.0(4.8)$ & $23.6(7.8)$ & .42 \\
SES (sd) & $30.2(13.1)$ & $37.1(13.0)$ & .09 \\
PSES (sd) & $43.8(13.7)$ & $49.5(8.1)$ & .11 \\
PANSSP & $21.1(4.8)$ & & \\
PANSSN & $17.6(5.3)$ & & \\
PANSST & $77.7(14.5)$ & & \\
SAPS & $1.7(0.7)$ & & \\
SANS & $2.0(0.6)$ & & \\
MEDS & $124.9(165.7)$ & &
\end{tabular}

SES, Socioeconomic status; PSES, Parental socioeconomic status; PANSSP, Positive and Negative Syndrome Scale positive factor score; PANSSN, PANSS negative factor score; PANSST, PANSS total score; SAPS, Scale for the Assessment of Positive Symptoms Averaged Global Scores; SANS, Scale for the Assessment of Negative Symptoms Averaged Global Scores; MEDS, Antipsychotic medication in chlorpromazine equivalents (oral dosages from Andreasen et al. (35), depot dosages from Gardner et al. (36).

\section{Neuropsychological Tests}

All participants completed the MATRICS Cognitive Consensus Battery and the WASI. Social functioning was assessed with the Global Assessment Scale (GAS), Global Functioning: Social and Role scales (GF:S, GF:R), the brief UCSD Performance-based Skills Assessment (UPSA-B) and the Social Functioning Scales (SFS, see Table 2 for neuropsychological test and social functioning scores).

\section{Procedure}

EEG was collected while subjects underwent two passive auditory tasks. Stimuli were generated with Tone Generator $(\mathrm{NCH}$ Software), and presented in Presentation (Neurobehavioral Systems, Inc.). Binaural auditory stimuli were presented at 80 $\mathrm{dB}$ using Etymotic $3 \mathrm{~A}$ insert earphones, with loudness confirmed with a sound meter. Participants watched a silent nature video while tones were played over earphones. They were asked to concentrate on the movie and ignore the tones.

\section{Stimuli}

\section{Simple MMN}

Stimuli comprised a standard tone $(1 \mathrm{kHz}, 50 \mathrm{msec}$ duration, 5 msec rise/fall), a pitch deviant $(1.2 \mathrm{kHz}, 50 \mathrm{msec}$ duration, 5 $\mathrm{msec}$ rise/fall), and a duration deviant $(1 \mathrm{kHz}, 100 \mathrm{msec}$ duration, $5 \mathrm{msec}$ rise/fall), presented with a stimulus onset asynchrony of $330 \mathrm{msec}$. A total of 1600 tones were presented, including 1,280 standards (80\%), 160 pitch deviants (10\%), and 160 duration deviants (10\%).

\section{Complex MMN}

Temporal proximity was used to form discrete groups of tones, with a SOA within groups of $330 \mathrm{msec}$ and an inter-trial interval of $750 \mathrm{msec}$. A total of 350 groups were presented. The standard group consisted of 3 identical tones ( 300 groups, $1 \mathrm{kHz}, 50 \mathrm{msec}$ duration, $5 \mathrm{msec}$ rise/fall, identical to the standard tone in the simple MMN task). The deviant groups (50 groups, 14.3\%) contained an additional fourth identical tone, and never immediately followed one another.

\section{EEG Recording}

EEG was recorded from a custom 72 channel Active2 high impedance system (BioSemi), comprising 70 scalp sites including the mastoids, 1 nose reference electrode, and 1 electrode below the right eye. The EEG amplifier bandpass was DC to $104 \mathrm{~Hz}(24 \mathrm{~dB} /$ octave rolloff) digitized at $512 \mathrm{~Hz}$, referenced to a common mode sense site (near PO1).

\section{MMN Analysis}

Processing was done off-line with EEGLAB (37) and BrainVision Analyzer2 (Brain Products GMBH). First, using EEGLAB, EEG was filtered at $0.5 \mathrm{~Hz}$ to remove DC drifts and skin potentials. Data were visually examined and any channels with excessive noise were interpolated. AMICA was used to remove one vertical and one horizontal EOG component.

Following pre-processing in EEGLAB, event-related potentials were processed using BrainVision Analyzer2. 
TABLE 2 | Neuropsychological and social functioning measures.

\begin{tabular}{|c|c|c|c|}
\hline & FESz & $\mathrm{HC}$ & $p$ \\
\hline WASI IQ & $108.1(14.7)$ & $106.3(8.8)$ & .62 \\
\hline MATRICS Speed & 45.5 (16.3) & $50.6(7.7)$ & .19 \\
\hline MATRICS AttVig & $41.6(12.0)$ & $48.4(6.3)$ & .025 \\
\hline MATRICS WM & $44.9(14.6)$ & 45.5 (10.2) & .86 \\
\hline MATRICS Verbal & $44.2(12.2)$ & $51.8(10.2)$ & .031 \\
\hline MATRICS Visual & $43.5(12.4)$ & $43.9(7.5)$ & .88 \\
\hline MATRICS Reason & $45.8(11.8)$ & $50.1(6.8)$ & .15 \\
\hline MATRICS SocCog & $43.1(11.2)$ & $54.1(5.9)$ & $<.001$ \\
\hline MATRICS Overall & $39.5(14.5)$ & $48.5(7.1)$ & .013 \\
\hline GAS & $35.6(8.9)$ & & \\
\hline UPSA-B Comm & $72.7(13.2)$ & & \\
\hline UPSA-B Finance & $82.6(14.8)$ & & \\
\hline UPSA-B Total & $77.7(9.7)$ & & \\
\hline GF : Role Current & $5.9(2.2)$ & $9.0(0.2)$ & $<.001$ \\
\hline GF : Role Highest & $7.7(1.2)$ & $9.0(0.0)$ & $<.001$ \\
\hline GF : Role Lowest & $5.7(2.2)$ & $9.0(0.2)$ & $<.001$ \\
\hline GF : Social Current & $5.4(1.8)$ & $9.0(0.2)$ & $<.001$ \\
\hline GF : Social Highest & $7.2(1.2)$ & $9.0(0.2)$ & $<.001$ \\
\hline GF : Social Lowest & $5.2(1.7)$ & $9.0(0.1)$ & $<.001$ \\
\hline SFS : Withdrawal & $100.6(11.1)$ & & \\
\hline SFS : Interpersonal & 121.77 (22.3) & & \\
\hline SFS : Recreational & 110.7 (11.8) & & \\
\hline SFS : Employment & $117.4(5.4)$ & & \\
\hline SFS : Independence-comp & $114.9(10.1)$ & & \\
\hline SFS : Independence-perf & $102.7(12.0)$ & & \\
\hline SFS : Pro-social & $109.7(10.8)$ & & \\
\hline
\end{tabular}

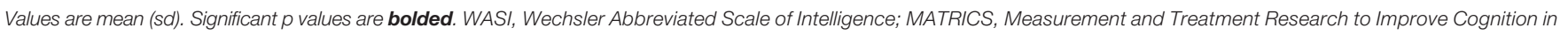

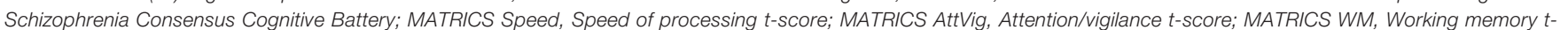

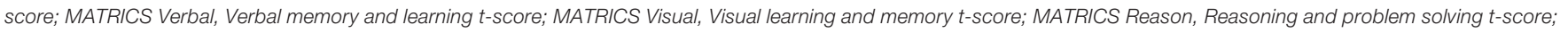

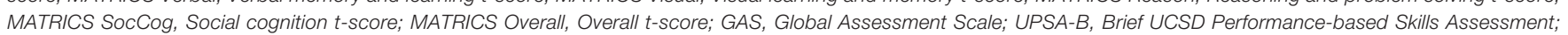

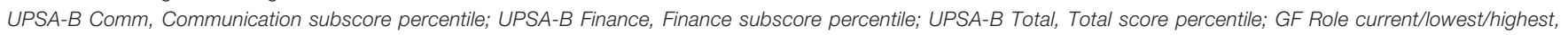

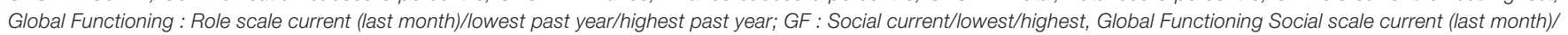

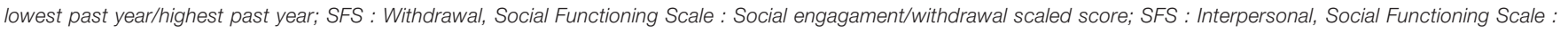

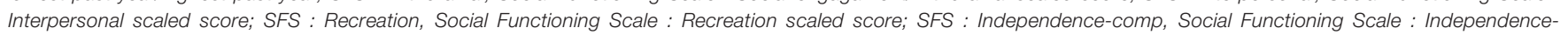

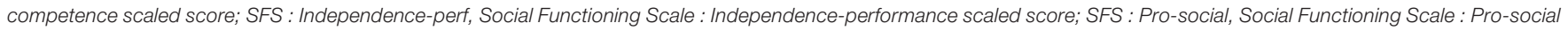
scaled score.

Data were low-pass filtered at $20 \mathrm{~Hz}$ to remove muscle and other high frequency artifact, and rereferenced to averaged mastoids.

\section{Simple MMN}

Epochs of $350 \mathrm{msec}$, including a $50 \mathrm{msec}$ prestimulus baseline, were extracted to deviant tones, and the standard tones preceding a deviant. Epochs were DC detrended between the first and last $50 \mathrm{msec}$, baseline corrected, and subsequently rejected if any site contained activity $\pm 50 \mu \mathrm{V}$. Averages were constructed for the standard tones preceding a deviant, pitch deviants, and duration deviants. Pitch MMN (pMMN) was visualized by subtracting the standard average from the pitch deviant average. Duration MMN (dMMN) was visualized by subtracting the standard average from the duration deviant average. MMN was measured by detection of the peak MMN at $\mathrm{FCz}$ in the group grand averages, and voltage then averaged over a 25 msec window centered on that latency for each individual at 6 frontocentral sites (F1, Fz, F2, FC1, FCz, FC2) where cMMN was largest (pMMN: FESz 95-120 msec; HC(FE) 95-120 msec; Sz 95-120 msec; HC(Sz) 97-122 msec, dMMN: FESz 158-183 msec; HC(FE) 162-187 msec; Sz 168-193 msec; $\mathrm{HC}(\mathrm{Sz})$ 156-181 $\mathrm{msec})$.

\section{Complex MMN}

Epochs of $750 \mathrm{msec}$ were extracted, including a $100 \mathrm{msec}$ prestimulus baseline. All epochs were DC detrended between the first and last $50 \mathrm{msec}$, baseline corrected, and subsequently rejected if any site contained activity $\pm 50 \mu \mathrm{V}$. Averages were constructed for the standard group last tones and the deviant group extra tones. cMMN was visualized by subtracting the standard group ending tone average from the deviant extra fourth tone average. MMN was measured by detection of the peak latency of the late cMMN at $\mathrm{FCz}$ in the group grand averages, and voltage then averaged over a $25 \mathrm{msec}$ window centered on peak latency for each individual at the 6 frontocentral sites. Due to the apparent early and late MMNs, two time periods were analyzed; early cMMN (130-155 msec for all groups), and late cMMN (430-455 msec for all groups).

\section{Analyses}

Group demographics and neuropsychological scores were compared using t-tests and chi-squared tests where appropriate. MMN analyses utilized repeated-measures ANOVA, with group (FESz, HC) as the between subjects factor, and electrode chain (F or FC) and site (left, central, or right) as within subjects factors. The Huynh-Feldt epsilon was used to correct for potential violations of sphericity of the site 
factor. Effect sizes were calculated as Cohen's d at FCz, the difference between the group means expressed in pooled standard deviations. Two-tailed Spearman's correlations were used to examine relationships between $\mathrm{MMN}$ at $\mathrm{FCz}$ and demographic, clinical, and neuropsychological items. Values are reported as Mean \pm SD. Significance was attained at $\mathrm{p}<.05$.

\section{RESULTS}

\section{Simple MMN}

pMMN was not reduced in FESz $(\mathrm{F} 1,42=0.2, \mathrm{p}=.657$, Figure 1). No other main effects or any interactions were significant. The effect size d for pMMN at FCz was 0.08 SD (See Table 3 for individual site voltages).

dMMN was not reduced in FESz $(\mathrm{F} 1,42=0.001, \mathrm{p}=.976$, Figure 2). dMMN was marginally larger along the FC chain than the $\mathrm{F}$ chain $(\mathrm{F} 1,42=3.62, \mathrm{p}=.064)$ and this distribution did not differ between groups. $\mathrm{dMMN}$ was larger at center and right sites than the left $(\mathrm{F} 2,84=8.93, \mathrm{p}=.001, \epsilon=0.80)$ and this distribution did not differ between groups. No remaining interactions were significant. The effect size $d$ for $d M M N$ at

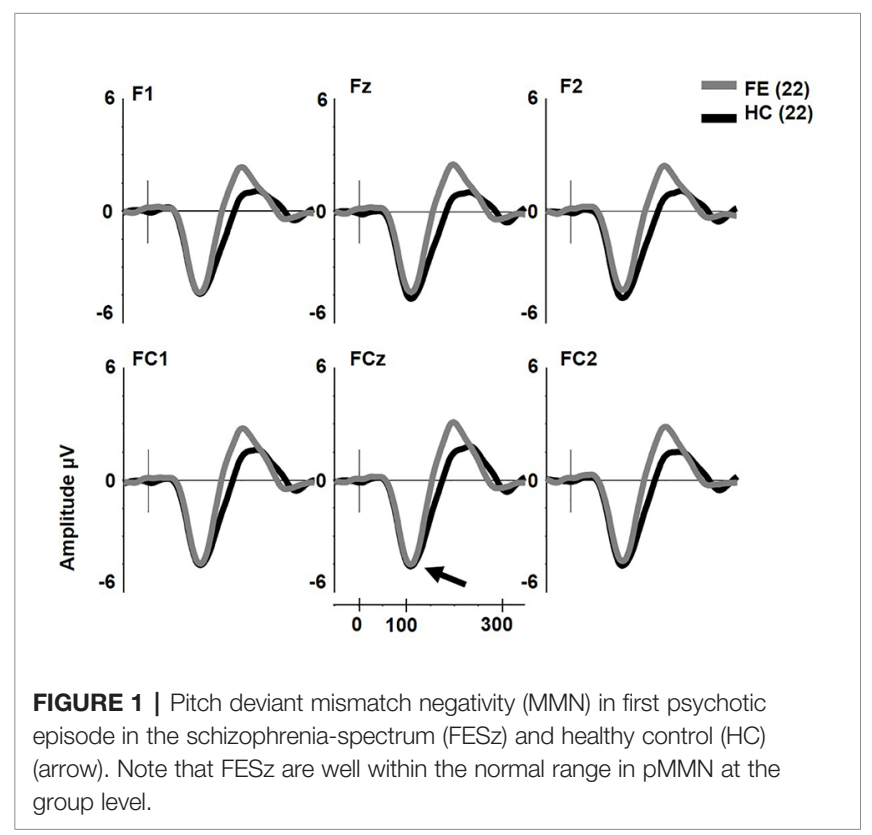

$\mathrm{FCz}$ was $-0.02 \mathrm{SD}$, indicating $\mathrm{FESz}$ were larger than $\mathrm{HC}$, albeit non-meaningfully (See Table 3 for individual site voltages).

\section{Extra-Tone Complex MMNs}

The early cMMN was not different between groups $(p>.2)$ and will not be discussed further. Unlike pMMN and $\mathrm{dMMN}$, which were not reduced, late cMMN (henceforth referred to as cMMN) was significantly impaired in FESz (FESz: $-0.1 \mu \mathrm{V}$, HCFE: -1.2 $\mu \mathrm{V} . \mathrm{F} 1,42=6.2, \mathrm{p}=.016$. Figure 3. See Table 3 for site voltages). The Cohen's d for group differences at FCz was $0.82 \mathrm{SD}$. No other main effects or interactions were significant.

\section{Neuropsychological and Clinical Correlations}

There were no significant correlations in FESz between cMMN amplitudes and WASI or MATRICS composite scores. Likewise, there were no significant correlations with PANSS total scores or positive, negative, or thought disorder factor scores. Among social functioning measures, larger $\mathrm{cMMN}$ was associated with higher SFS independence-performance scaled scores $(r=-.54$, $\mathrm{p}=.009$, Figure 4). cMMN was uncorrelated with duration of untreated psychosis (DUP).

\section{DISCUSSION}

In this sample of individuals at first contact with psychiatric services for psychosis, with only a short course of antipsychotic medication, sMMN was not reduced at the group level, with a small effect size for both pMMN $(\mathrm{d}=0.08)$ and dMMN $(\mathrm{d}=-0.02)$. By contrast, the later cMMN to a pattern deviant, in this case an extra $4^{\text {th }}$ tone among groups of 3 tones, revealed a significant and large deficit $(\mathrm{d}=0.82)$. Thus, the results are consistent with the hypothesis that the increased computational demand of pattern processing as reflected in cMMN by contrast with the relatively easily discriminable physical parameter changes eliciting sMMN is more sensitive to the earlier and more subtle pathophysiology during the early course of the progressive pathology in schizophrenia. Although the exact neural correlates of the cMMN are unknown, it is assumed the same circuits process novelty for sMMN and $\mathrm{cMMN}$ within the prediction error scheme (38), and likely rely on the same NMDAr mediated circuit plasticity (39). This may be a promising framework for development of a biological circuit-based approach to new

TABLE 3 | Simple mismatch negativity (sMMN) and complex MMN (CMMN) values.

\begin{tabular}{|c|c|c|c|c|c|c|}
\hline & F1 & Fz & F2 & FC1 & FCz & FC2 \\
\hline \multicolumn{7}{|c|}{ pMMN } \\
\hline FESz & $-4.2(1.7)$ & $-4.1(1.7)$ & $-4.0(1.7)$ & $-4.2(1.7)$ & $-4.2(1.7)$ & $-4.1(1.7)$ \\
\hline HC & $-4.3(1.6)$ & $-4.5(1.6)$ & $-4.5(1.7)$ & $-4.3(1.8)$ & $-4.4(1.9)$ & $-4.4(2.0)$ \\
\hline \multicolumn{7}{|c|}{ dMMN } \\
\hline FESz & $-3.2(1.8)$ & $-3.3(1.8)$ & $-3.4(1.8)$ & $-3.3(1.9)$ & $-3.5(1.9)$ & $-3.5(1.8)$ \\
\hline HC & $-3.2(2.4)$ & $-3.4(2.2)$ & $-3.4(2.3)$ & $-3.4(2.5)$ & $-3.5(2.7)$ & $-3.5(2.6)$ \\
\hline \multicolumn{7}{|c|}{ cMMN } \\
\hline FESz & $-0.1(1.6)$ & $-0.1(1.5)$ & $-0.2(1.4)$ & $0.0(1.5)$ & $0.0(1.8)$ & $-0.2(1.4)$ \\
\hline $\mathrm{HC}$ & $-1.1(1.6)$ & $-1.1(1.6)$ & $-1.3(1.6)$ & $-1.2(1.5)$ & $-1.4(1.7)$ & $-1.3(1.6)$ \\
\hline
\end{tabular}

Values are mean (SD). Values are in $\mu \mathrm{V}$. 


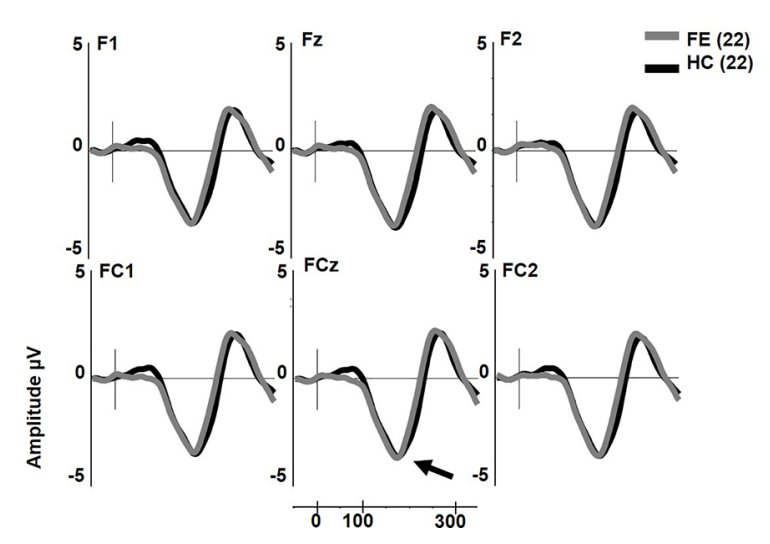

FIGURE 2 | Duration deviant mismatch negativity (MMN) in first psychotic episode in the schizophrenia-spectrum (FESz) and healthy control $(\mathrm{HC})$ (arrow). Note that FESz are well within the normal range in $\mathrm{pMMN}$ at the group level.

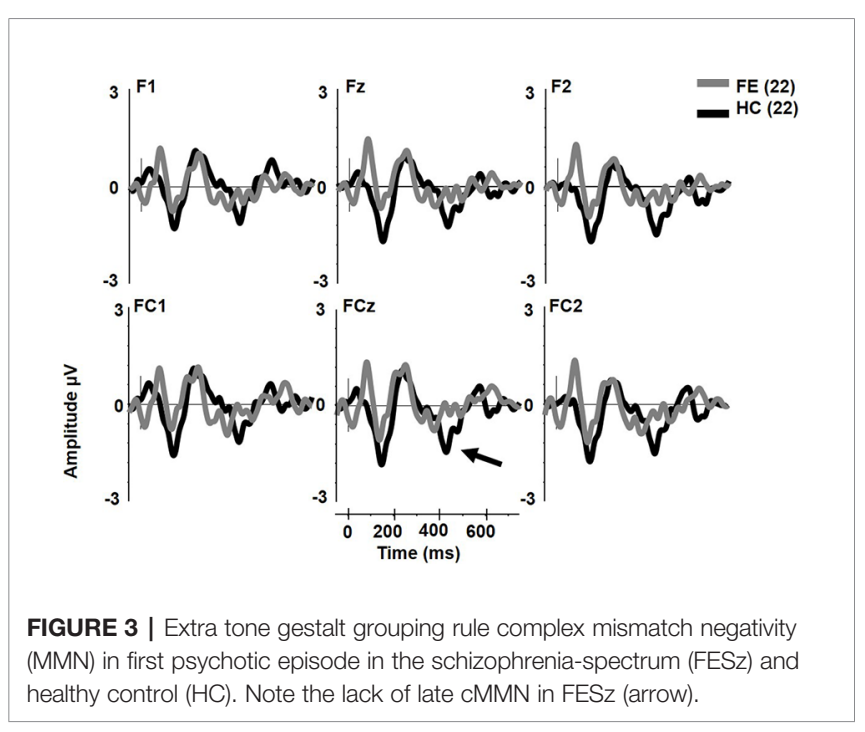

pharmacologic interventions, but the present data do not allow extrapolation to the circuit or synaptic level. Future sourceresolved human studies and invasive animal work may shed light on the regional and circuit similarities and differences in the cortical substrate of cMMN and sMMN.

cMMN was not significantly different from zero in FESz. Hence, it is not entirely clear if correlations are valid, in the sense that the cMMN may only reflect neurophysiological noise. However, FESz did show a range of cMMN amplitudes (Figure 4), and those with larger $\mathrm{cMMN}$ tended to have better utilization of social skill sets. While cMMN has not been correlated with social functioning in FESz previously, we have shown that $\mathrm{CMMN}$ correlated with emotional withdrawal, poor attention, and lack of spontaneity and flow of conversation in long term schizophrenia (23). The strong association between reduced cMMN and impaired utilization of available social skills is consistent with the original reports of reduced social

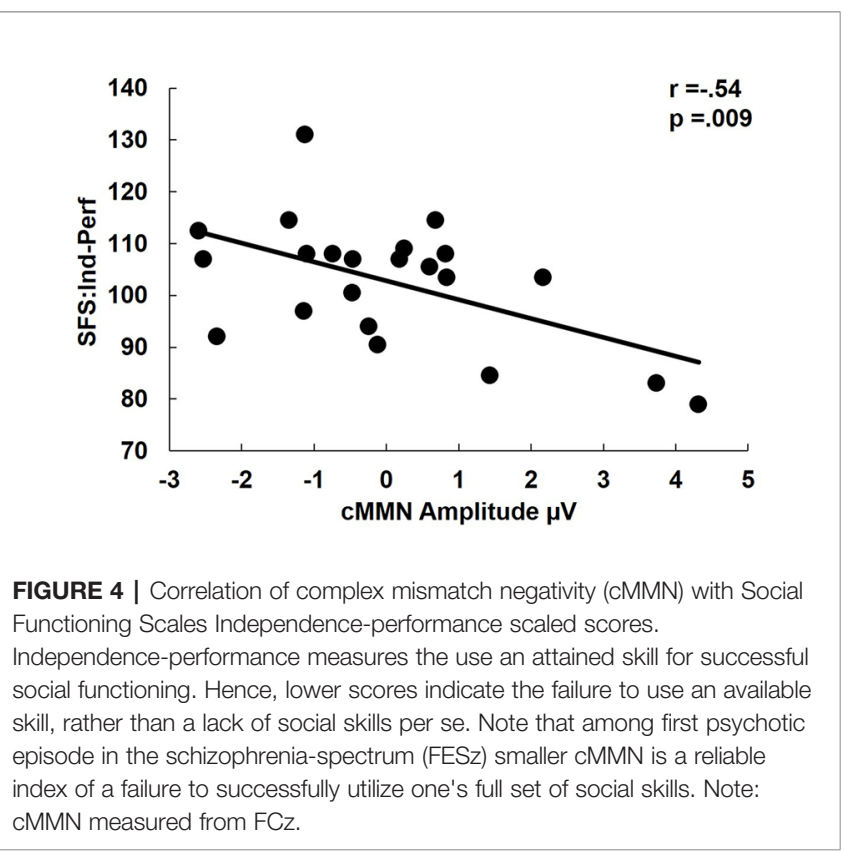

functioning being associated with $\mathrm{dMMN}$ in long term schizophrenia, e.g., (40). Still, given the relatively restricted range of $\mathrm{cMMN}$ this finding would need to be replicated and investigated further for meaningful interpretation.

The fact that cMMN was not significantly different from zero in FESz argues strongly for its utility as a biomarker of disease presence. This is even more compelling in the context of an essentially normal pMMN and $\mathrm{dMMN}$ in the same FESz subjects that showed a markedly impaired cMMN. Thus, it appears that pattern-deviance tasks that necessitate a higher level of processing may be more sensitive to subtle auditory cortex pathology than relatively simple changes in a single acoustic feature. One might speculate that the degree of pathology needed to impair detection of pure tone differences is rather substantial. cMMN appears to be sensitive to more modest pathology. Future studies should compare first episode schizophrenia-spectrum to affective-spectrum individuals to determine the specificity of the cMMN deficit on this task.

Although CHR individuals were not tested, we suggest a measure with a large deficit in FESz may be useful for investigation of CHR individuals. While all CHR individuals may show general auditory pathology, it is possible that the cMMN deficits emerge only in those that are in the true prodrome to schizophrenia. While no single test is likely to be diagnostic, the extra tone cMMN appears to be an important tool that when used in conjunction with other biological, neuropsychological, and clinical measures can provide an objective indication of risk for transition to psychosis. It is important to note that most "risk calculators" for transition to psychosis and studies of factors predisposing to eventual conversion are based on clinical and psychological rather than biological aspects of presentation. For example, Cannon et al. (41) found 5 factors contributed to risk for transition to psychosis: genetic risk with deterioration in functioning, 
greater paranoia and unusual thought content, worse social functioning, and a history of substance abuse. Cannon et al. (42) reported that greater paranoia and unusual thought content, greater decline in social functioning, poor verbal learning and memory, slow speed of processing, and younger age at clinical contact contributed to risk for transition to psychosis. Ruhrmann et al. (43) developed a prediction model based on schizotypal disorder, positive symptoms, bizarre thinking, sleep disturbances, decline in social functioning in the last year, and years of education. Fusar-Poli et al. (44) have developed an online calculator for the transition to psychosis among individuals accessing secondary mental health care (not the general population), based on presumptive diagnosis, age, sex, sex by age, and ethnicity. None of these calculators include a biological measure.

By contrast with the clinically-based risk calculators, a growing number of studies explored baseline and longitudinal measures useful in predicting group membership once transition to psychosis occurred (or more correctly over a followup period such as 2, 3, or 5 years). In their recent meta-analysis, Studerus, Ramyead, and Riecher-Rössler (45) indicated 91 studies that developed or validated a prediction model for the transition to psychosis. Among those studies, only 10 used EEG measures, 7 used MRI measures, and 5 used blood-based biomarkers. Thus, objective biological tests that may indicate the transition to psychosis are a critical unmet need for early intervention. We do not know if cMMN will be a useful biomarker before the onset of psychosis, but its reduction at first psychosis with a large effect size is encouraging. Future work needs to examine the possibility in CHR individuals that those in a true psychosis prodrome show selective cMMN reduction. This presupposes that the same auditory pathophysiology is common across various etiologies to psychosis. As described earlier, the fact that most individuals in the early and long-term phases of schizophrenia show MMN reduction suggests it is a systems-level pathology that cuts across specific etiologies for schizophrenia.

Several issues remain unanswered. Although the effect size for cMMN reduction was large $(\mathrm{d}=0.82)$, the samples were relatively modest, and the results must be replicated. Because this is the first application of the extra tone cMMN task at first episode, and there are relatively few cMMN studies in psychosis, the reliability of the measure is unknown. The cMMN was small in $\mathrm{HC}$, which may limit its utility. Currently, we are developing new pattern MMN paradigms to elicit larger $\mathrm{cMMN}$ in $\mathrm{HC}$, which presumably will increase the group separation. We do not know whether the cMMN and sMMN share the same biological substrate, particularly given the longer latency of cMMN. Neither do we know what the distributed neural system underlying cMMN is. Relatedly, we do not know whether cMMN is dependent on NMDAr function. Future work must resolve these molecular issues. It is not known what $\mathrm{cMMN}$ looks like in CHR. A robust decrement in $c M M N$ at first episode in the lack of sMMN reduction indicates that the probability of cMMN providing useful information about subtle underlying neural pathophysiology in true prodromal cases among CHR individuals is greater than sMMN. Still, it remains unknown. Currently, we are testing this paradigm in such individuals.

In summary, we showed that cMMN to an extra tone among frequent groups of standard size was reduced in FESz, while pMMN and dMMN were not. Further the cMMN correlated with social functioning, with worse functioning associated with smaller cMMN. These novel data provide the first step in development of a MMN-based task that is more sensitive to the subtle pathology early in the course of schizophrenia. We speculate that $\mathrm{cMMN}$ may be a useful probe of pathophysiology in the true psychosis prodrome.

\section{DATA AVAILABILITY STATEMENT}

The raw data supporting the conclusions of this article will be made available by the authors, without undue reservation, to any qualified researcher.

\section{ETHICS STATEMENT}

The studies involving human participants were reviewed and approved by University of Pittsburgh Institutional Review Board. The patients/participants provided their written informed consent to participate in this study.

\section{AUTHOR CONTRIBUTIONS}

DS provided substantial contributions to the conception and design of the work; the acquisition, analysis, and interpretation of data for the work; and drafting the work and revising it critically for important intellectual content. BC provided substantial contributions to the acquisition, analysis, and interpretation of data for the work, and revising the report critically for important intellectual content. SH provided substantial contributions to the acquisition, analysis, and interpretation of data for the work, and revising the report critically for important intellectual content. All authors provided approval for publication of the content, and agree to be accountable for all aspects of the work in ensuring that questions related to the accuracy or integrity of any part of the work are appropriately investigated and resolved.

\section{FUNDING}

This work was supported by NIH R01 MH094328 to DS.

\section{ACKNOWLEDGMENTS}

We thank Timothy K. Murphy, BS, Kayla Ward, BS, and Justin R. Leiter-McBeth, BA, for data acquisition and analysis, and the faculty and staff of the WPIC Psychosis Recruitment and 
Assessment Core, the Conte Center for Translational Mental Health Research (P50 MH103204, David Lewis, MD, Director), and the University of Pittsburgh Clinical Translational Science

\section{REFERENCES}

1. Umbricht D, Krljez S. Mismatch Negativity in Schizophrenia: A MetaAnalysis. Biol Psychiatry (2005) 76:1-23. doi: 10.1016/j.schres.2004.12.002

2. Erickson MA, Ruffle A, Gold JM. A meta-analysis of mismatch negativity in schizophrenia: from clinical risk to disease specificity and progression. Biol Psychiatry (2016) 79:980-7. doi: 10.1016/j.biopsych.2015.08.025

3. Salisbury DF, Polizzotto NR, Nestor PG, Haigh SM, Koehler J, McCarley RW. Pitch and duration MMN and premorbid intellect in the first hospitalized schizophrenia spectrum. Schizophr Bull (2017) 43:407-16. doi: 10.1093/ schbul/sbw074

4. Salisbury DF, Shenton ME, Griggs CB, Bonner-Jackson A, McCarley RW. Mismatch negativity in chronic schizophrenia and first-episode schizophrenia. Arch Gen Psychiatry (2002) 59:686-94. doi: 10.1001/ archpsyc.59.8.686

5. Salisbury DF, Kuroki N, Kasai K, Shenton ME, McCarley RW. Progressive and interrelated functional and structural evidence of post-onset brain reduction in schizophrenia. Arch Gen Psychiatry (2007) 64:521-9. doi: 10.1001/archpsyc.64.5.521

6. Magno E, Yeap S, Thakore JH, Garavan H, De Sanctis P, Foxe JJ. Are auditory-evoked frequency and duration mismatch negativity deficits endophenotypic for schizophrenia? High-density electrical mapping in clinically unaffected first-degree relatives and first-episode and chronic schizophrenia. Biol Psychiatry (2008) 64:385-91. doi: 10.1016/ j.biopsych.2008.03.019

7. Haigh SM, Coffman BA, Salisbury DF. Mismatch negativity in first-episode schizophrenia: a meta-analysis. Clin EEG Neurosci (2017) 48:3-10. doi: $10.1177 / 1550059416645980$

8. Bodatsch M, Ruhrmann S, Wagner M, Müller R, Schultze-Lutter F, Frommann I, et al. Prediction of psychosis by mismatch negativity. Biol Psychiatry (2011) 69:959-66. doi: 10.1016/j.biopsych.2010.09.057

9. Perez VB, Woods SW, Roach BJ, Ford JM, McGlashan TH, Srihari VH, et al. Automatic auditory processing deficits in schizophrenia and clinical high-risk patients: forecasting psychosis risk with mismatch negativity. Biol Psychiatry (2014) 75:459-69. doi: 10.1016/j.biopsych.2013.07.038

10. Atkinson RJ, Michie PT, Schall U. Duration mismatch negativity and P3a in first-episode psychosis and individuals at ultra-high risk of psychosis. Biol Psychiatry (2012) 71:98-104. doi: 10.1016/j.biopsych.2011.08.023

11. Atkinson RJ, Fulham WR, Michie PT, Ward PB, Todd J, Stain H, et al. Electrophysiological, cognitive and clinical profiles of at-risk mental state: The longitudinal Minds in Transition (MinT) study. PloS One (2017) 12: pe0171657. doi: 10.1371/journal.pone.0171657

12. Bruggemann JM, Stockill HV, Lenroot RK, Laurens KR. Mismatch negativity (MMN) and sensory auditory processing in children aged 9-12 years presenting with putative antecedents of schizophrenia. Int J Psychophysiol (2013) 89:374-80. doi: 10.1016/j.ijpsycho.2013.05.008

13. Javitt DC, Steinschneider M, Schroeder CE, Arezzo JC. Role of cortical Nmethyl-D-aspartate receptors in auditory sensory memory and mismatch negativity generation: implications for schizophrenia. Proc Natl Acad Sci (1996)93:11962-7. doi: 10.1073/pnas.93.21.11962

14. Paavilainen $P$, Jaramillo $M$, Näätänen R. Binaural information can converge in abstract memory traces. Psychophysiology (1998) 35:483-7. doi: 10.1017/ S0048577298970895

15. Paavilainen P, Jaramillo $M$, Näätänen R, Winkler I. Neuronal populations in the human brain extracting invariant relationships from acoustic variance. Neurosci Lett (1999) 265:179-82. doi: 10.1016/S0304-3940(99)00237-2

16. Tervaniemi M, Radil T, Radilova J, Kujala T, Näätänen R. Pre-attentive discriminability of sound order as a function of tone duration and interstimulus interval: a mismatch negativity study. Audiol Neurotol (1999) 4:303-10. doi: 10.1159/000013854

17. Gumenyuk V, Korzyukov O, Alho K, Winkler I, Paavilainen P, Näätänen R. Electric brain responses indicate preattentive processing of abstract acoustic
Institute (UL1 RR024153, Steven E. Reis, MD) for their assistance in recruitment, diagnostic and psychopathological assessments, and neuropsychological evaluations of participants.

regularities in children. Neuroreport (2003) 14:1411-5. doi: 10.1097/ 00001756-200308060-00001

18. Korzyukov OA, Winkler I, Gumenyuk VI, Alho K. Processing abstract auditory features in the human auditory cortex. Neuroimage (2003) 20:2245-58. doi: 10.1016/j.neuroimage.2003.08.014

19. van Zuijen TL, Simoens VL, Paavilainen P, Näätänen R, Tervaniemi M. Implicit, intuitive, and explicit knowledge of abstract regularities in a sound sequence: an event-related brain potential study. J Cogn Neurosci (2006) 18:1292-303. doi: 10.1162/jocn.2006.18.8.1292

20. Alain C, Hargrave R, Woods DL. Processing of auditory stimuli during visual attention in patients with schizophrenia. Biol Psychiatry (1998) 44:1151-9. doi: 10.1016/S0006-3223(97)00478-2

21. Paavilainen P, Arajärvi P, Takegata R. Preattentive detection of nonsalient contingencies between auditory features. Neuroreport (2007) 18:159-63. doi: 10.1097/WNR.0b013e328010e2ac

22. Salisbury DF. Finding the missing stimulus mismatch negativity: Emitted MMN to violations of an auditory gestalt. Psychophysiology (2012) 49:544-8. doi: 10.1111/j.1469-8986.2011.01336.x

23. Haigh SM, Coffman BA, Murphy TK, Butera CD, Salisbury DF. Abnormal Auditory Pattern Perception in Schizophrenia. Schizophr Res (2016) 176:4739. doi: 10.1016/j.schres.2016.07.007

24. Korpilahti P, Krause CM, Holopainen I, Lang AH. Early and Late Mismatch Negativity Elicited by Words and Speech-Like Stimuli in Children. Brain Lang (2001) 76:332-9. doi: 10.1006/brln.2000.2426

25. Zachau S, Rinker T, Körner B, Kohls G, Maas V, Hennighausen K, et al. Extracting rules: early and late mismatch negativity to tone patterns. Neuroreport (2005) 16:2015-9. doi: 10.1097/00001756-200512190-00009

26. Grimm S, Escera C. Auditory deviance detection revisited: Evidence for a hierarchical novelty system. Int J Psychophysiol (2012) 85:88-92. doi: 10.1016/ j.ijpsycho.2011.05.012

27. Escera C, Malmierca MS. The auditory novelty system: an attempt to integrate human and animal research. Psychophysiology (2014) 51:111-23. doi: 10.1111/ psyp. 12156

28. Recasens M, Grimm S, Wollbrink A, Pantev C, Escera C. Encoding of nested levels of acoustic regularity in hierarchically organized areas of the human auditory cortex. Hum Brain Mapp (2014) 35:5701-16. doi: 10.1002/hbm.22582

29. Salisbury DF, McCathern AG. Abnormal complex auditory pattern analysis in schizophrenia reflected in an absent missing stimulus mismatch negativity. Brain Topography (2016) 29:867-74. doi: 10.1007/s10548-016-0514-2

30. Rudolph ED, Ells EML, Campbell DJ, Abriel SC, Tibbo PG, Salisbury DF, et al. Finding the missing-stimulus mismatch negativity (MMN) in early psychosis: Altered MMN to violations of an auditory gestalt. Schizophr Res (2015) 166:158-63. doi: 10.1016/j.schres.2015.05.028

31. Salisbury DF, McCathern AG, Coffman BA, Murphy TK, Haigh SM. Complex mismatch negativity to tone pair deviants in long-term schizophrenia and in the first-episode schizophrenia spectrum. Schizophr Res (2018) 191:18-24. doi: 10.1016/j.schres.2017.04.044

32. Haigh SM, De Matteis M, Coffman BA, Murphy TK, Butera CD, Ward KL, et al. Mismatch negativity to pitch pattern deviants in schizophrenia. Eur J Neurosci (2017) 46:2229-39. doi: 10.1111/ejn.13660

33. Cornblatt BA, Lencz T, Smith CW, Correll CU, Auther AM, Nakayama E. The schizophrenia prodrome revisited: a neurodevelopmental perspective. Schizophr Bull (2003) 29:633-51. doi: 10.1093/oxfordjournals.schbul.a007036

34. Cornblatt BA, Carrión RE, Addington J, Seidman L, Walker EF, Cannon TD, et al. Risk factors for psychosis: impaired social and role functioning. Schizophr Bull (2012) 38:1247-57. doi: 10.1093/schbul/sbr136

35. Andreasen NC, Pressler M, Nopoulos P, Miller D, Ho BC. Antipsychotic dose equivalents and dose-years: a standardized method for comparing exposure to different drugs. Biol Psychiatry (2010) 67(3):255-62.

36. Gardner DM, Murphy AL, O'Donnelll H, Centorrino F, Baldessarini RJ. International consensus study of antipsychotic dosing. Focus (2014) 12 (2):235-43. 
37. Delorme A, Makeig S. EEGLAB: an open source toolbox for analysis of singletrial EEG dynamics including independent component analysis. J Neurosci Methods (2004) 134:9-21. doi: 10.1016/j.jneumeth.2003.10.009

38. Winkler I. Interpreting the mismatch negativity. J Psychophysiol (2007) 21:147-63. doi: 10.1027/0269-8803.21.34.147

39. Wacongne C, Changeux JP, Dehaene S. A neuronal model of predictive coding accounting for the mismatch negativity. J Neurosci (2012) 32:3665-78. doi: 10.1523/JNEUROSCI.5003-11.2012

40. Light GA, Braff DL. Mismatch Negativity Deficits Are Associated with Poor Functioning in Schizophrenia Patients. Arch Gen Psychiatry (2005) 62:12736. doi: 10.1001/archpsyc.62.2.127

41. Cannon TD, Cadenhead K, Cornblatt B, Woods SW, Addington J, Walker E, et al. Prediction of psychosis in youth at high clinical risk: a multisite longitudinal study in North America. Arch Gen Psychiatry (2008) 65:28-37. doi: 10.1001/archgenpsychiatry.2007.3

42. Cannon TD, Yu C, Addington J, Bearden CE, Cadenhead KS, Cornblatt $\mathrm{BA}$, et al. An individualized risk calculator for research in prodromal psychosis. Am J Psychiatry (2016) 173:980-8. doi: 10.1176/appi.ajp.2016. 15070890

43. Ruhrmann S, Schultze-Lutter F, Salokangas RK, Heinimaa M, Linszen D, Dingemans $\mathrm{P}$, et al. Prediction of psychosis in adolescents and young adults at high risk: results from the prospective European prediction of psychosis study.
Arch Gen Psychiatry (2010) 67:241-51. doi: 10.1001/archgenpsychiatry. 2009.206

44. Fusar-Poli P, Rutigliano G, Stahl D, Davies C, Bonoldi I, Reilly T, et al. Development and validation of a clinically based risk calculator for the transdiagnostic prediction of psychosis. JAMA Psychiatry (2017) 74:493500. doi: 10.1001/jamapsychiatry.2017.0284

45. Studerus E, Ramyead A, Riecher-Rössler A. Prediction of transition to psychosis in patients with a clinical high risk for psychosis: a systematic review of methodology and reporting. psychol Med (2017) 47:1163-78. doi: $10.1017 /$ S0033291716003494

Conflict of Interest: The authors declare that the research was conducted in the absence of any commercial or financial relationships that could be construed as a potential conflict of interest.

Copyright (๑) 2020 Salisbury, Coffman and Haigh. This is an open-access article distributed under the terms of the Creative Commons Attribution License (CC $B Y$ ). The use, distribution or reproduction in other forums is permitted, provided the original author(s) and the copyright owner(s) are credited and that the original publication in this journal is cited, in accordance with accepted academic practice. No use, distribution or reproduction is permitted which does not comply with these terms. 